\title{
A biomimetic platform for studying root-environment interaction
}

\author{
Pallavi Kumari • Neta Ginzburg • Tali Sayas • Sigal Saphier • Patricia Bucki • \\ Sigal Brown Miyara • Denise L. Caldwell • Anjali S. Iyer-Pascuzzi • Maya Kleiman (iD
}

Received: 28 June 2019 / Accepted: 9 December 2019/Published online: 13 December 2019

(C) The Author(s) 2019

\begin{abstract}
Aims Microstructure plays an important role in biological systems. Microstructural features are critical in the interaction between two biological organisms, for example, a microorganism and the surface of a plant. However, isolating the structural effect of the interaction from all other parameters is challenging when working directly with the natural system. Replicating microstructure of leaves was recently shown to be a powerful research tool for studying leaf-environment interaction. However, no such tool exists for roots. Roots present a special challenge because of their delicacy (specifically
\end{abstract}

Responsible Editor: Peter J. Gregory of root hairs) and their 3D structure. We aim at developing such a tool for roots.

Methods Biomimetics use synthetic systems to mimic the structure of biological systems, enabling the isolation of structural function. Here we present a method which adapts tools from leaf microstructure replication to roots. We introduce new polymers for this replication. Results We find that Polyurethane methacrylate (PUMA) with fast UV curing gives a reliable replication of the tomato root surface microstructure. We show that our system is compatible with the pathogenic soilborne bacterium Ralstonia solanacearum.

\section{P. Bucki · S. B. Miyara}

Institute of Plant Protection, Agricultural Research Organization (Volcani Center), 7505101 Rishon Lezion, Israel

\section{P. Bucki}

e-mail: pbucki@volcani.agri.gov.il

\author{
P. Kumari \\ e-mail: palcuj31@gmail.com \\ N. Ginzburg \\ e-mail: neta.livne88@gmail.com \\ T. Sayas \\ e-mail: talid@volcani.agri.gov.il
}

S. Saphier

Department of Organic Chemistry, Israel Institute for Biological Research, 74100 Ness-Ziona, Israel

e-mail: sigals@iibr.gov.il

\section{S. B. Miyara}

e-mail: sigalhor@volcani.agri.gov.il

D. L. Caldwell · A. S. Iyer-Pascuzzi

Department of Botany and Plant Pathology, Purdue University, West Lafayette, IN 47907, USA

\section{L. Caldwell}

e-mail: denisecaldwell74@gmail.com
A. S. Iyer-Pascuzzi
e-mail: asi2@purdue.edu 
Conclusions This newly developed tool may be used to study the effect of microstructure, isolated from all other effects, on the interaction of roots with their environment.

Keywords Root Surface $\cdot$ Microstructure $\cdot$ Synthetic . Biomimetics $\cdot$ Method $\cdot$ Interaction

\section{Introduction}

The first point of contact in the interaction between plants and their surroundings is the surface of the plant. This interaction is composed of two components: a physical one (due to the surface microstructure) and a chemical/molecular one (due to molecular signals expressed on the surface). When studying plant surface interaction with its environment using the natural plant, it is impossible to separate those two components as they are entangled together within the biological system. An example of this is found in plant roots. Roots are vital to plant health, as they are the plant's source of water and nutrient uptake and provide anchorage in the soil. In addition to these functions, plant roots need a robust immune system, because they must defend themselves against a daily barrage of soil pathogens such as bacteria, fungi and nematodes. These microbes invade the root, multiply, and can ultimately destroy the plant. The soil microbes first point of contact will always be the surface of the root. Although root structure has been studied in the past with great emphasis on the topology and architecture of the root system (Mancuso 2012; Smith and De Smet 2012) the root microstructure has been rarely addressed and mostly in the context of root hairs and their assistance in nutrient absorbance from the soil (Leitner et al. 2010; Marschner 2011). The unique influence of root microstructure on root-microorganism interaction has never been tested.

Biomimetics is an emerging field at the interface of biological and material sciences. Biomimetics takes inspiration from nature and mimics natural structures in synthetic systems to address human problems (Bhushan 2009). Plant structures and surfaces have always been a point of inspiration (Barthlott et al. 2017). One well known example is the microstructure of the super hydrophobic lotus leaf (Koch et al. 2010; Solga et al. 2007). The development of biomimetic artificial super hydrophobic surfaces was motivated by various practical applications, such as anticorrosive, antifogging, oil spill clean-up, oil/water separation, and drag reduction (Liu et al. 2007; Weng et al. 2011; Yang et al. 2012; Yang et al. 2018). Various methods have been reported to produce artificial super hydrophobic surfaces, including electrodeposition, hydrothermal, sol-gel, soft lithography and others (Lai et al. 2009; Lau et al. 2003; Thieme et al. 2001; Tricinci et al. 2015; Zhao et al. 2007; Zhao et al. 1997). One of those methods, nanolithography, including photo/chemical/soft lithography, is a quick and easy method for generating surface structure replications (Rogers and Nuzzo 2005). Among the different techniques, soft lithography has attracted considerable interest given its potential for fabricating a variety of two- and three-dimensional architectures, with materials and surface properties that are biocompatible with multiple applications. Surface microstructural features can be replicated at up to $100-500 \mathrm{~nm}$ resolution to create larger uniform patterns (Zhang et al. 2011). The popularity of soft lithography for replica molding is manifest in the growing number of publications from the end of the twentieth century. The most widely used material for soft lithography is the biocompatible, transparent, cheap, commercially available silicone elastomer polydimethylsiloxane (PDMS) (Mark et al. 2005). PDMS is widely used in the field of microelectromechanical systems (MEMS) as well as in the fluid flow field, generating microfluidic devices (Anderson et al. 2000; Kleiman et al. 2015; Mata et al. 2005; Schneider et al. 2009). PDMS is easy to manipulate and can be formed into multiple shapes mostly using photo/chemical/soft lithography (Rogers and Nuzzo 2005).

Biomimetics is usually used as a synthetic platform to mimic biological structures for the use of mankind. Additionally, biomimetics has also proven to be a useful research tool to study how microstructure influences microorganism-plant interactions. Using both photo lithography for a mask, to generate specific leaf structural features (such as stomata and trichomes) and soft lithography to replicate the entire leaf microstructure (Dasgupta et al. 2015; Doan and Leveau 2015; Sirinutsomboon et al. 2011), recent work has demonstrated that different structural features influence the interaction of the leaf with macro organisms such as bed bugs (Szyndler et al. 2013) and microorganisms like E. coli (Zhang et al. 2014). Using this method, leaf trichomes were shown to puncture the legs of bedbugs, and bacteria were shown to prefer specific structural features for colonization. In one study, agar was used in addition to PDMS, showing that the PDMS can 
accept other additives (Zhang et al. 2014). Despite advances with leaf surface microstructure, the application of biomimetics to root surfaces has lagged behind. One reason for this may be because certain surface structures such as root hairs are very delicate (usually more delicate than trichomes) and hence are difficult to replicate using PDMS based soft lithography.

In this method paper, we show the first replication of the root surface microstructure, soft lithography. We use tomato (Solanum lycopersicum) as our model system, and emphasize root hairs. Soft lithography is challenging for root surface replication and hence we report our strategy to overcome the difficulties using different materials and our success with one of those materials. We selected three different molding materials: Gelatin-gel, Polydimethylsiloxane (PDMS), and UV curable gel Polyurethane methacrylate (PUMA) (Zhao et al. 1997). All materials were chosen due to biocompatibility, affordability and easy processability. Unlike PDMS that received extensive exploration in the field of material sciences as a molding material, both gelatin gel (Paretkar et al. 2014; Rogers and Nuzzo 2005) and PUMA (Kerai et al. 2015) received very little attention in the context of lithography and biomimetics. We used these three materials to obtain templates (negatives) with different physical characteristics: templates made by gelatin gel were prepared with high concentrations of plasticizer and were very soft while the PUMA templates formulation showed rigid structure and high mechanical strength. PDMS is a soft elastomer that gave an intermediate platform between the soft Gelatin and the rigid PUMA. We found that the molding material PUMA mimics the microstructure of the original template in a highly faithful manner. We developed the template using a soft lithography strategy combined with a UVcuring technique (Peng et al. 2013). Finally, we prepared the positive replication (using the PUMA mold - negative template) from PDMS, giving the first reported replication of root surface microstructure in a synthetic material. Additionally, we show the feasibility of our method as a tool to study root surface-microorganism interaction. We show that Ralstonia solanacearum (Rs), a soilborne pathogenic bacterium of tomato that colonizes plant roots, is differently distributed on a structured and a non-structured root replica. We believe that these materials might be of interest for the fabrication of biotemplate microstructures of biological components for various applications, including studies of the role of microstructure in root-environment interactions.

\section{Materials and method}

1. Materials: Gelatin powdered porcine skin was purchased from Glentham LIFE SCIENCES, United Kingdom. Glycerol anhydrous was purchased from Bio-lab ltd. SYLGARD ${ }^{\text {TM }} 184$ Silicon Elastomer Base, and SYLGARD ${ }^{\mathrm{TM}} 184$ Silicon Elastomer Curing agent were purchased from Dow chemicals, United States. All other chemicals were purchased from Sigma Aldrich.

2. Plant materials: Root samples were all taken from Solanum lycopersicum M82 cultivar. Roots were grown in two distinct protocols. In the first option, plants were grown in soil. After four weeks, the plant was removed from soil, the root system was cut, the plant was kept in water for a week and new, adventitious roots grown from the stem, were taken for viewing and replication. In the second process, M82 seeds were germinated on a wet Whatman paper in a petri dish, in a growing room at $25^{\circ} \mathrm{C}$. Whatman paper was kept moist for a duration of 7 days, after which the growing roots germinating from the seed were detached from the seed. The section about $0.5 \mathrm{~cm}$ from the seed was used for replica generation.

3. Replication protocol: All root replication protocols were based on a previously established leaf surface replication protocol. The protocol for leaf synthetic microstructure replication is described in Scheme 1 and is performed by a technique called soft lithography. The process requires two steps that first involves the molding of a leaf surface by a liquid polymer that later cures to form a mirror image (negative replica) of the leaf surface microstructure. This negative replica goes through a functionalization process to avoid the adhesion of the next layer, as described in point 5 of this section. At this point, an additional molding is performed, this time using the negative replica as the source, to generate the replication (positive replica) of the surface microstructure.

4. Preparation of Gelatin negative replica: Gelatin -gel was prepared from gelatin powder. Gelatin powder was dissolved in distilled water at $10 \% \mathrm{w} /$ $\mathrm{w}$ and stirred at $60{ }^{\circ} \mathrm{C}$ for $1 \mathrm{~h}$ until complete dispersion of gelatin powder in water. Glycerol was then added at $45 \% \mathrm{w} / \mathrm{w}$ and the solution was stirred for an additional $2 \mathrm{~h}$ at $60{ }^{\circ} \mathrm{C}$. The solution was then left at $60{ }^{\circ} \mathrm{C}$ with no stirring for an 


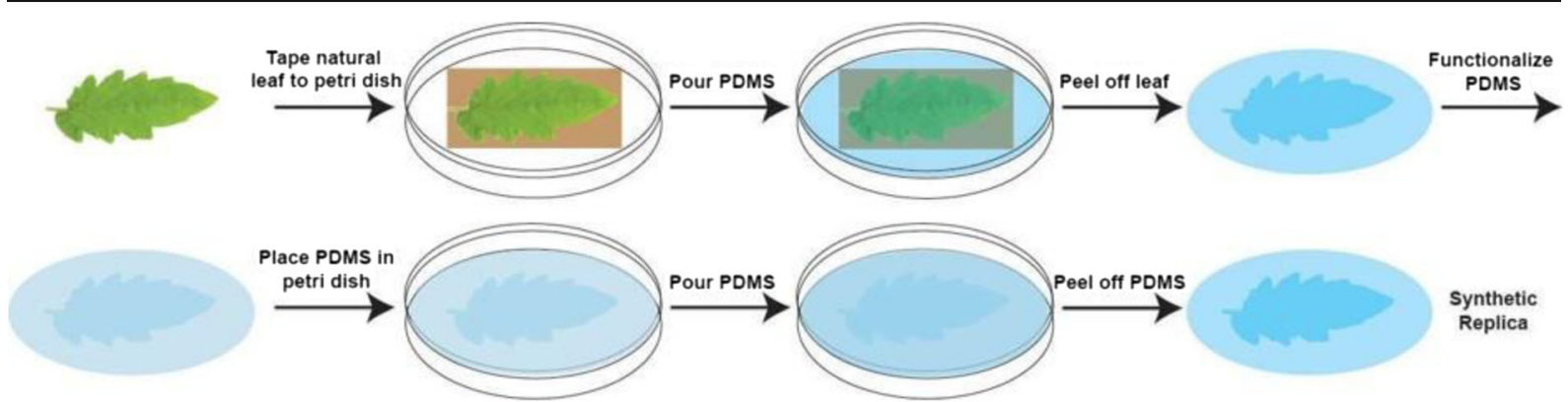

Scheme 1 The process for making the biomimetic leaf replica. A natural leaf is taped to a petri dish. Liquid Polydimethylsiloxane (PDMS) is poured and vacuum is applied to assure good coverage of the microstructure. Polymer is cured overnight at room temperature. Leaf is then peeled off from the cured polymer to generate the negative replica. The negative replica is functionalized to turn

additional hour to remove air bubbles. This resulted in a homogeneous, transparent solution. The root was put on a petri dish and the solution was applied on top of it and left at room temperature (RT) for $4 \mathrm{~h}$. After a gel was formed, the root was removed by forceps, obtaining a gelatin gel template with root surface microstructure mirror image (negative replica). This negative replica was only visualized via light microscopy.

5. Preparation of PDMS negative replica: Sylgard 184 polymer kit was used. Prepolymer and curing agent were well mixed at a $10 / 1 \mathrm{w} / \mathrm{w}$ ratio of polymer/curing agent and then kept under vacuum for $1 \mathrm{~h}$ to remove air bubbles. The natural root was taped to a petri dish and the polymer solution was poured on top of the root. Vacuum was applied for $2 \mathrm{~h}$ to assure full coverage of the surface microstructure. The covered root was kept at room temperature overnight. The root was then peeled off the cured polymer, leaving the PDMS template of the root surface microstructure mirror image (negative replica). The negative replica could then be coated with a thin layer of gold for SEM visualization. To avoid attachment of the positive replica onto this negative replica, a functionalization process was performed. A BD-20 AC laboratory CORONA treater was used for a few seconds to activate the surface of the negative replica. The negative replica was then immediately placed in a desiccator with $100 \mu \mathrm{l}$ of Trichloro $(1 \mathrm{H}, 1 \mathrm{H}, 2 \mathrm{H}, 2 \mathrm{H}-$ perfluorooctyl) silane for $3 \mathrm{~h}$. The negative replica was then wrapped in aluminum foil and kept at RT until further use. We did not observe any time limitation for using this negative replica. the polymer hydrophilic, and prevent sticking of the two layers. The negative replica is then placed in a petri dish. Liquid polymer solution is poured on top of the negative replica, vacuumed for $2 \mathrm{~h}$ and cured at RT overnight. The negative replica is removed from the newly formed polymer layer, which represents the synthetic positive replica

\section{Preparation of Polyurethane negative replica:} For the formation of negative Polyurethane (PU) template we used a solution of diurethane dimethacrylate (Monomer 85\% w/w) and ethyl methacrylate $(15 \% \mathrm{w} / \mathrm{w})$ to which we added Diethyl Pathalate (Plasticizer 30\% w/w) for flexibility and a photo initiator (2-hydroxy-2methylpropiophenone) for polymerization (2-3\% $\mathrm{w} / \mathrm{w})$. All components were continuously stirred overnight at RT until a homogeneous solution was obtained. We then applied the homogeneous mixture above a root placed on a microscope slide. The slide was subjected to ultraviolet light ( 8 watt for $5 \mathrm{~min}$ ) for polymerization and film formation. The surface of the film was then rinsed with ethanol to remove unreacted monomers. We obtained a transparent polymeric film with a mirror image of root microstructure (negative replica). The polymer template was removed from the slide by scalpel and coated with a thin layer of gold for SEM observation.

7. Preparation of positive replica: After the formation of a negative replica by each material, the negative replica was used to generate the positive replica that mimics the microstructure of the root surface. We placed the negative replica in a petri dish, poured liquid PDMS (10:1, as was described previously), vacuumed (for $2 \mathrm{~h}$ ) and cured (at RT, overnight). We then removed the negative replica from the newly formed polymer layer to achieve the replication of the root surface microstructure (positive replica). This replica was then coated with a thin gold layer for SEM visualization. 
8. Preparation of a needle replica: As one of the controls, we prepared a replication of a needle in a similar diameter to that of the root. Since the needle surface is flat, we used PDMS as our molding material for both negative and positive replica. Preparation of the needle replica repeated steps 4 (preparation of PDMS negative replica) and 6 (preparation of positive replica) in this section only using a needle, rather than a root, as the template.

9. Root fixation: Roots were fixed using an increasing ethanol concentration, in a standard protocol for root visualization. Roots were placed in a solution of $70 \%$ ethanol (in water) overnight. Solution was then replaced to $80 \%, 90 \%$ and $100 \%$ of ethanol with incubation time of $1 \mathrm{~h}$ each. Solution was then changed again to a fresh $100 \%$ ethanol solution, in which the root was incubated overnight. Then, the root was dried on K850 critical point drier and coated with gold-palladium alloy in Q150T ES turbo-pumped sputter coater, following manufacturers' instructions (Quorum Technologies Ltd., UK).

10. Visualization of root and replica: Visualization of both root and replica were performed on both a wide field microscope and a Scanning Electron Microscopy (SEM). For SEM observation, the root was first fixed as described above. The replicas were coated with a thin gold layer as a preparation for SEM visualization. Wide field images were taken using Leica DM LB microscope using bright field with a Nikon DS-Fil camera. SEM images were taken using Jeol IT100 (Japan) 20KV WD10.

11. Rs work: Ralstonia solanacearum strain K60 was grown on casamino acid-peptone-glucose (CPG) agar with 2\% 2,3,5-triphenyl tetrazolium chloride (TZC) at $28{ }^{\circ} \mathrm{C}$ for $48 \mathrm{~h}$. CPG + TZC agar is commonly used for $R s$ growth. A single colony was selected from the agar and placed into CPG broth with $2 \%$ TZC and allowed to grow overnight to an optical density at $600 \mathrm{~nm}=0.1\left(1 \times 10^{8} \mathrm{CFU} /\right.$ $\mathrm{ml})$. This level of inoculum was chosen because it is commonly used in experiments which assess $R s$ - root colonization and resistance of soil-grown tomatoes to Rs (Caldwell et al. 2017; French et al. 2018). To 'inoculate' the synthetic surfaces, the needle replica and root replica were placed in CPG broth containing $R s$ at $10^{8} \mathrm{CFU} / \mathrm{ml}$ and placed on an orbital shaker in a $28{ }^{\circ} \mathrm{C}$ incubator overnight. In the morning the replicas were transferred to a 12 well plate and the inoculum was replaced. Inoculum was replaced to prevent toxic effects associated with bacterial overgrowth. As preparation for SEM, that evening the inoculum was removed from the wells and replaced with $100 \%$ ethanol by gently pipetting it into the well away from the replicas. After five minutes the ethanol was removed and the replicas were transferred to a $25 \mathrm{~mm}$ nickel stub. The surface of the stub was lined with carbon conductive adhesive tape upon which the replicas were placed. The stub was then placed in a closed box and placed in a $37{ }^{\circ} \mathrm{C}$ incubator overnight to dry. Samples were sputter coated for $90 \mathrm{~s}$ in a Cressington $208 \mathrm{HR}$ sputter coater with platinum to a thickness of $5 \mathrm{~nm}$. Samples were imaged on a NovaNano SEM 200 by FEI with a spot size of 3.0, working distance of $4.9 \mathrm{~mm}$ and a high voltage of $5.00 \mathrm{kV}$.

\section{Results}

The synthetic surface replication of leaf microstructural features has proven to be a very useful tool for studying the interaction between leaves and microorganisms, as it enabled isolation of structural elements in the interaction. We hypothesized that synthetic surface replicas would also benefit studies of root-environment interactions. Our goal was to mimic the microstructure of the root epidermis (the root surface) and use this mimic as a tool to study the effect of structural features on the interactions of the root surface and its environment, both living and inanimate.

As a first step it was important to observe the microstructure we were aiming to mimic - the root surface microstructure. To achieve this, we visualized two different types of roots by Scanning Electron Microscopy (SEM). We used adventitious roots growing from the stem of 2 week old plants after the removal of a root system (as described in the methods) as well as 7 day old roots germinating from a seed (as described in the methods). Both roots were taken from the M82 tomato cultivar. We observed different structures in both root types (Fig. 1). The adventitious root was mostly lacking hairs (Fig. 1A), while the seed germinated root had hairs at different densities and different length based on the location (Fig. 1B). This result was somewhat expected 

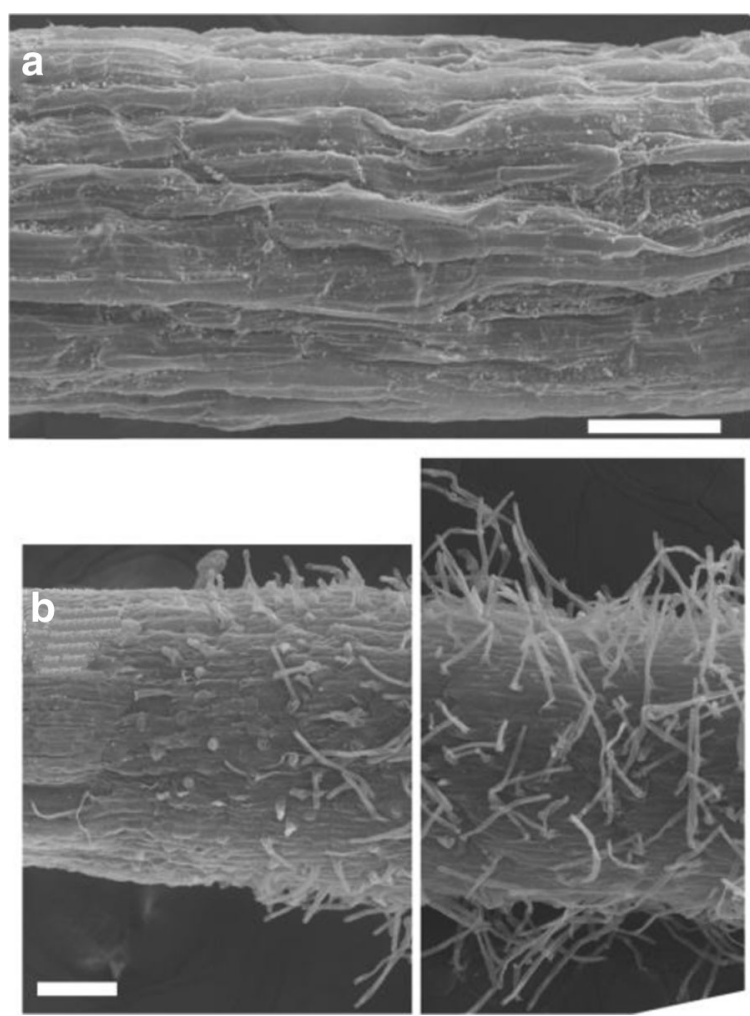

Fig. 1 SEM images of M82 tomatoes root surface A. Surface of a root grown from the stem of a 2 week old plant after the removal of the root system. No hairs are visible. B. Surface of a 7 day old root germinated from a seed on a wet Whatman paper. Hairs at different length are visible depending on the area of the root. Roots were fixed using ethanol followed by a critical point drying (CPD) and coated with a gold thin layer. (Scale bars $=100 \mu \mathrm{m}$ )

as, like other plants, primary roots of tomato are known to have a high density of root hairs (which might aid in penetration and nutrient and water initial uptake) (Tsai et al. 2004). In contrast, adventitious roots in other species have a varied amount of root hairs and some are claimed to not have any root hairs at all (Cormack 1949; Popham and Henry 1955). The cell structure also varied between the two root types and the locations at which the images were taken.

Our initial approach for mimicking the root surface structure was to copy the protocol that has been well established in the field of leaf surface synthetic microstructure replication (biomimetics) (Koch et al. 2009; materials and methods).

To establish a protocol for root surface structure replication we adjusted some of the parameters in the leaf surface structure replication protocol. The replication of the root surface structure was significantly more challenging than that of the leaf. Roots are curved and much more delicate than leaves, especially the root hairs. Taping the root to the petri dish was challenging. In addition it was challenging to avoid shrinkage and destruction of the biological tissue. We varied vacuum times and temperatures, tried different taping methods and different root pre-treatments, including fixing the root prior to the replication process. To obtain an optimal protocol in which the structure most similar to root surface microstructure was achieved, we examined the resulting replicas using SEM. The best results were obtained using the protocol described in the methods section. The results for this replication are shown in Fig. 2. However, unlike the replication process for leaf surface microstructure, we found that this replication process is far from perfect and we cannot replicate some important structural features, specifically the root hairs.

Due to the problems we encountered in replicating the root surface microstructure using the same materials and techniques used for leaf surface microstructure replication, we looked for different routes. Since we saw that after the preparation of the negative replica, using PDMS, the root we used as a template was dried out and the hairs were torn, we speculated that the main challenge was working with the delicate biological tissue, i.e. the root and preventing its complete destruction. For the preparation of the negative replica, we decided to use different materials that would prevent the destruction of the root and prevent the hairs from being torn. Our materials of choice are described in Scheme 2, which describes the whole process starting from the negative replica made from the biological tissue to the positive replica made from the negative one. As PDMS is a soft elastomer, we decided to proceed trying two opposite approaches. The first, using a much softer, biocompatible material based on an aqueous (gel) solution, in the hopes that the root will not be dried out in this environment during the negative replication. We also hypothesized that the removal of the root from a softer negative replica would be gentler and hence preserve root hair structure. For this option we chose the material gelatin with glycerol as a plasticizer (Scheme 2). The second option used a much tougher material that is a viscous liquid and enables a liquid environment. We hypothesized that the root would not dry out during the preparation and that the curing process would be sufficiently fast for a good replication of the root surface structure. Additionally, we hypothesized that the tough material might enable better removal of the root and would assist in avoiding root hair breakage, which 

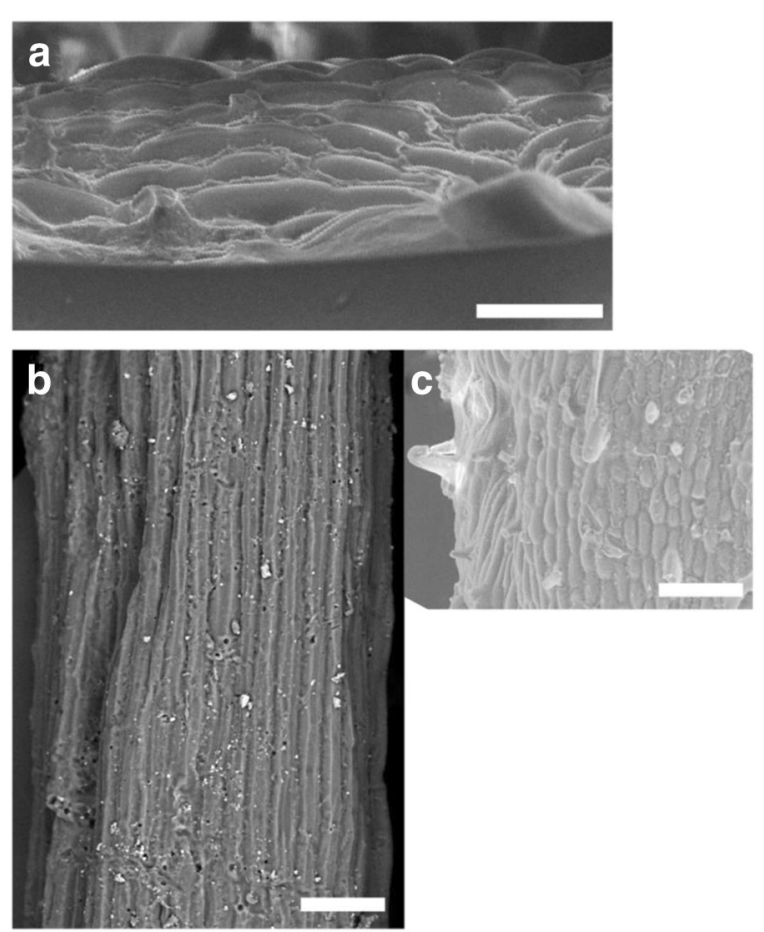

Fig. 2 SEM images of M82 tomato root surface replica made from PDMS negative A. Negative of the replication of surface of a 7 day old root germinated from a seed on a wet Whatman paper, made from PDMS (scale bar $=50 \mu \mathrm{m}$ ). B and C. Positive replication of surface of a root grown from the stem of a 2 week old plant after the removal of the root system (B) or a 7 day old root germinated from a seed on wet Whatman paper (C). (B) shows the typical structure of this root type surface. (C) shows the cell structure on the surface but fails to replicate the root hairs. Both positives are made from PDMS, using a PDMS negative mold. (scale bars $=100 \mu \mathrm{m}$ )

might damage the replication if the hairs stay in the synthetic material. The material of choice for this option was polyurethane modified with methacrylate at the edges - Polyurethane (methyl acrylate) (PUMA) (Scheme 2).

Using our softer material (Gelatin) we prepared the negative replica as described in the materials and methods section. Since the negative replica was made of Gelatin, which has a high concentration of water, it was impossible to take a SEM image of this negative replica without completely destroying it. Such an image will not give a reliable representation of the replica. Hence, a light microscopy image of this negative replica was taken (Fig. 3A). This turned out to be very challenging since the replica was very soft and flexible, preventing good focus. Therefore, the best option to check the reliability of our replication was to further proceed to the positive replication made of PDMS using the Gelatin negative replication as our mold. Those positive replicates were then viewed using SEM (Fig. 3B and C). We found that, as suspected from the light microscope images, the gelatin is a poor platform for this type of replication. We could not replicate the root hairs nor could we replicate any of the surface features of the root. In fact, the gelatin performed even more poorly than the PDMS (Fig. 3). While we thought this formulation would ease the separation between the negative replica and the root, we found that the separation was incomplete, probably due to the stickiness of the Gelatin. In fact, when removing the root from the Gelatin based negative replica, we sometimes witnessed residues of the Gelatin matrix on the removed root. Many times, those residues were not detected by the naked eye, and were only seen using a microscope. We suspect that this unclean separation prevented the replication of the surface microstructure by the Gelatin negative replica and caused a destruction of the microstructure as represented by this negative replica. The separation between the PDMS positive replica and the gelatin negative replica was of a significantly higher quality and hence this stage probably does not represent the failing point. In an attempt to optimize this process, we used different amounts of the plasticizer, glycerol, for the preparation of the Gelatin solutions, from $0,10,20$ and $30 \%$ and up to the $45 \%$ reported here. The less plasticizer we used the less soft the material was, surprisingly, addition of plasticizer did not contribute much to the flexibility of the material. In all plasticizer concentrations, and varying softness degrees, the separation between the Gelatin and the root was unclean. Hence, we attribute this to a characteristic of the Gelatin itself and not to the softness of the formed gel. Possibly, there is an interaction between the gelatin and the root that prevents clean separation.

We then moved to the more rigid polymer: Polyurethane methacrylate (PUMA). We prepared a negative replica from PUMA as described in the materials and methods section, using the great advantage of fast (10 min) curing under UV light. The strength of the polymer made the separation between the polymer and the replica challenging, as we needed to capture the root and gently remove it from the replica without tearing it. Unlike the case with the Gelatin replica, the separation between the root and the PUMA replica left no visible residues of root in the replica, or polymer on root. This enabled us to remove the whole root with the root hairs attached, 


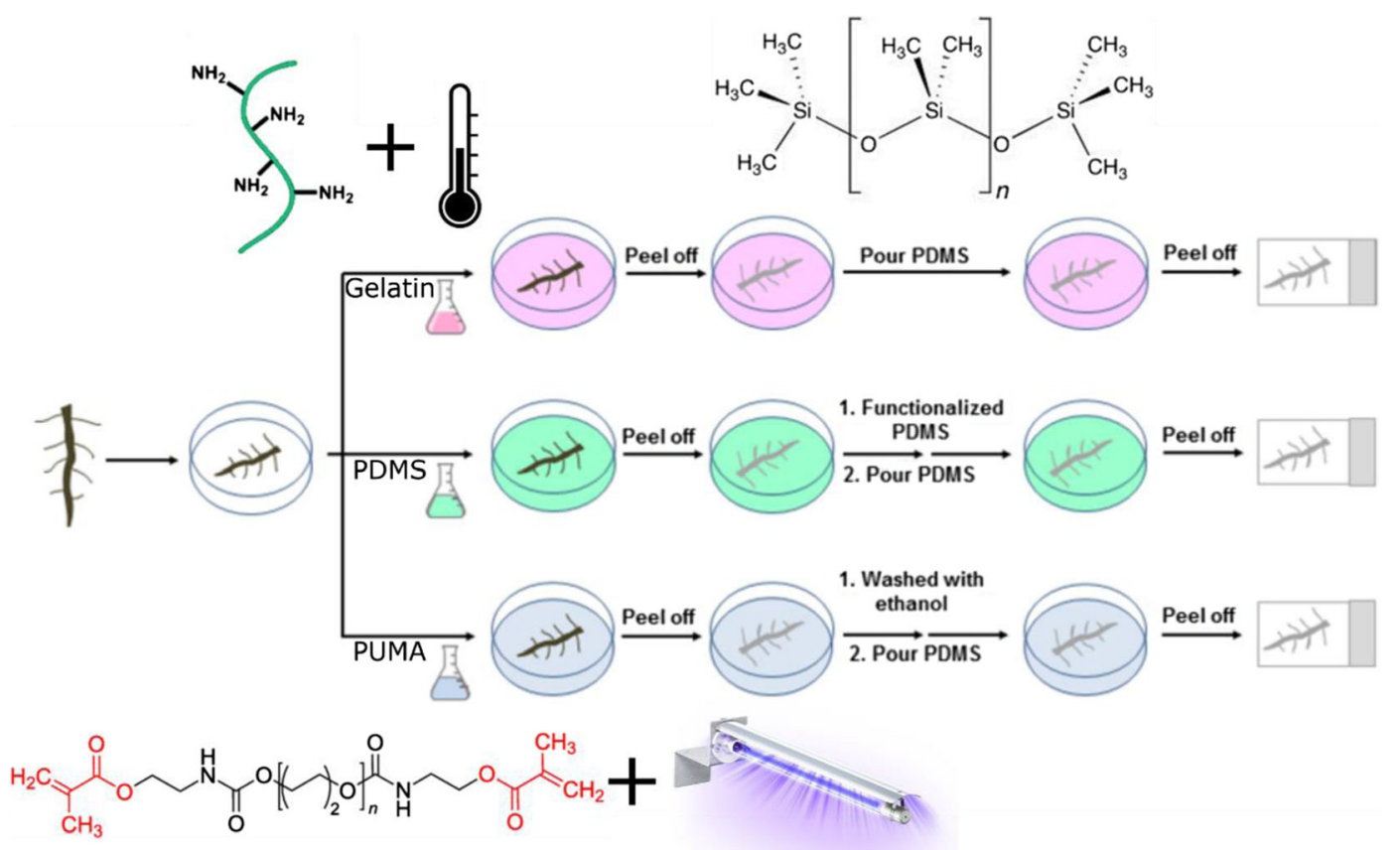

Scheme 2 Schematic diagram for the preparation of positive replicas of M82 tomato root made from three different molding materials. We prepared the solutions as described in the materials and methods section. The three solutions (Gelatin, PDMS and PUMA), were cured to form a negative replica of the root surface structure. Gelatin solution was prepared using heat (top left of image shows gelatin structure). PUMA solution was

leaving behind a perfect replication (Fig. 4A). We used this negative replica to generate positive replicas using PDMS as before. In the replica made from roots germinated from seeds on Whatman paper, where root hairs were present, we managed to replicate the root hairs in our PDMS replica (Fig. 4B). In the replica made from the adventitious roots, where root hairs were missing, the replica reproduced the cell structure on the surface of the root at a very high resolution (Fig. 4C). It is very hard to quantitatively compare the natural root and the replica. As this study was aimed at presenting our ability to replicate the general known root features (specifically root hairs), we made sure we can see those general features (cell structure and root hairs) with no quantitative analysis between identical roots. The results obtained using this replication method show that a replication of root surface microstructure using a revised soft lithography method is possible. Hence cured using UV light (PUMA structure is shown in image bottom left). The negative templates were obtained by direct casting of the prepared solution on the root surface. The obtained negative template was covered with PDMS solution followed by vacuum and curing at room temperature to form the positive replica. All positive replicas were made from PDMS, the structure of which is shown in the image top this method can be used as a tool to examine the effect of surface microstructure in root-environment interaction.

For successful studies of structure function, exact structural replication is necessary yet not sufficient. It is critical that we also evaluate whether the synthetic platform can be used as a tool to study interactions between the root and its environment. To do that we chose Ralstonia solanacearum $(R s)$ as a model. Tomato is highly susceptible to this soilborne bacterial pathogen (Nion and Toyota 2015; Kim et al. 2016), which is the causal agent of bacterial wilt disease. To cause disease, soilborne bacterial pathogens like $R s$ must first attach to the root surface. Root attachment is critical for disease, and $R s$ mutants that cannot attach to the root surface have decreased virulence (Kang et al. 2002; Yang et al. 2013; Dalsing and Allen 2014). After root attachment, $R s$ enters the root through minor wounds or cracks in the root surface that occur as the root grows through soil and 

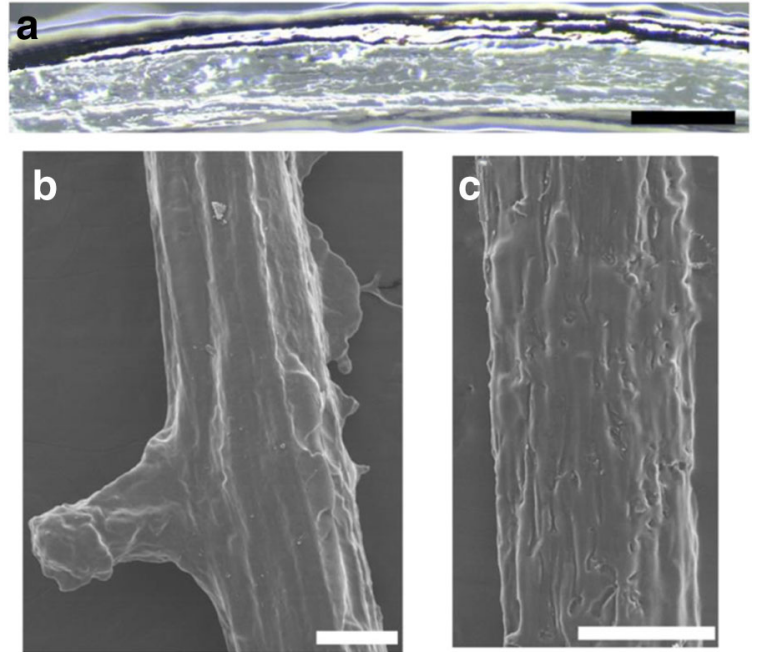

Fig. 3 images of M82 tomato root surface replicas from Gelatin negative. A. Light microscopy image of negative surface replica of a 2 week old plant after the removal of the root system, made from Gelatin. (scale bar $=500 \mu \mathrm{m}$ ). B and C. SEM images of a positive replication of surface of the same root type. (B) shows the emergence of a lateral root. (C) shows a different area of the root surface which shows similarities to the root surface structure, but with very poor replication. Both positives were made from PDMS and using a Gelatin negative mold. Both positives were coated by a thin layer of gold prior to visualization. (scale bars = $200 \mu \mathrm{m})$

when lateral roots emerge from the root. Once inside the root, the pathogen eventually enters the root vasculature where it further multiplies. Mechanisms of resistance to $R s$ in crop species are not well understood, and whether structural features on the root surface play a role in tomato resistance to $R s$ is unknown. To test the feasibility of our synthetic system to aid in understanding the attachment of Rs to root surface structures, we inoculated synthetic PDMS surfaces with Rs. The surfaces were dipped in a $10^{8} \mathrm{CFU} / \mathrm{ml}$ solution of bacteria, dried, fixed and viewed using SEM. The inoculation was performed on two types of surfaces: a structureless surface which used a needle as the mold for replication (Fig. 5, D-F), and a structured surface which replicates a root (Fig. 5 A-C). The bacteria show a distinct pattern of colonization on the two chemically identical but structurally different surfaces (Fig. 5). We can see that while the distribution of bacteria on the structureless surface looks random, the distribution on the structured surface shows preference to micro-valleys of the root surface topography. This result shows that our system might aid in explaining root-microorganism interaction from a strictly structural perspective.

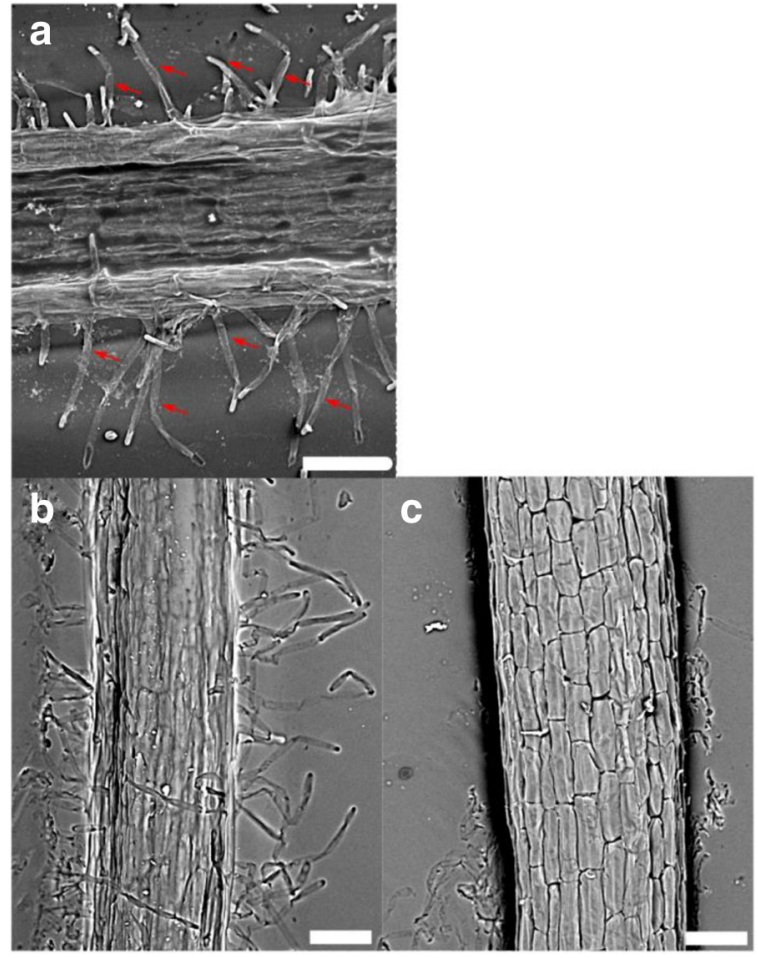

Fig. 4 SEM images of M82 tomato root surface replica from PUMA negative. A. Negative of the replication of surface of a 7 day old root germinated from a seed on a wet Whatman paper, made from PUMA. The voids made by root hairs are clearly shown (marked by red arrows). B and C. Positive replication of $\mathrm{s}$ surface of the same root type (B) or a root grown from the stem of a 2 week old plant after the removal of the root system (C). (B) shows the cell structure on the surface and replicates the root hairs. (C) shows the typical structure of this root type surface, with very distinct cell structures. Both positives are made from PDMS and using a PUMA negative mold. Both positives and the negative were coated by a thin layer of gold prior to visualization. (scale bars $=100 \mu \mathrm{m}$ )

\section{Discussion}

The interface between biology and material sciences has great potential to enrich material science and engineering as well as our understanding of natural biological systems. Surface microstructure greatly influences a microorganism's behavior (Bhattacharjee et al. 2017; Bixler and Bhushman 2012; Gule et al. 2016). Exploring the influence of surface microstructure in the context of a biological system could shed new light on interactions between the biological surface and the microorganism. In previous studies of interactions between biological surfaces and microorganisms, the structural role has been mostly absent (Diem 1974; Lindsey and Pugh 1976). This is understandable since this role is 

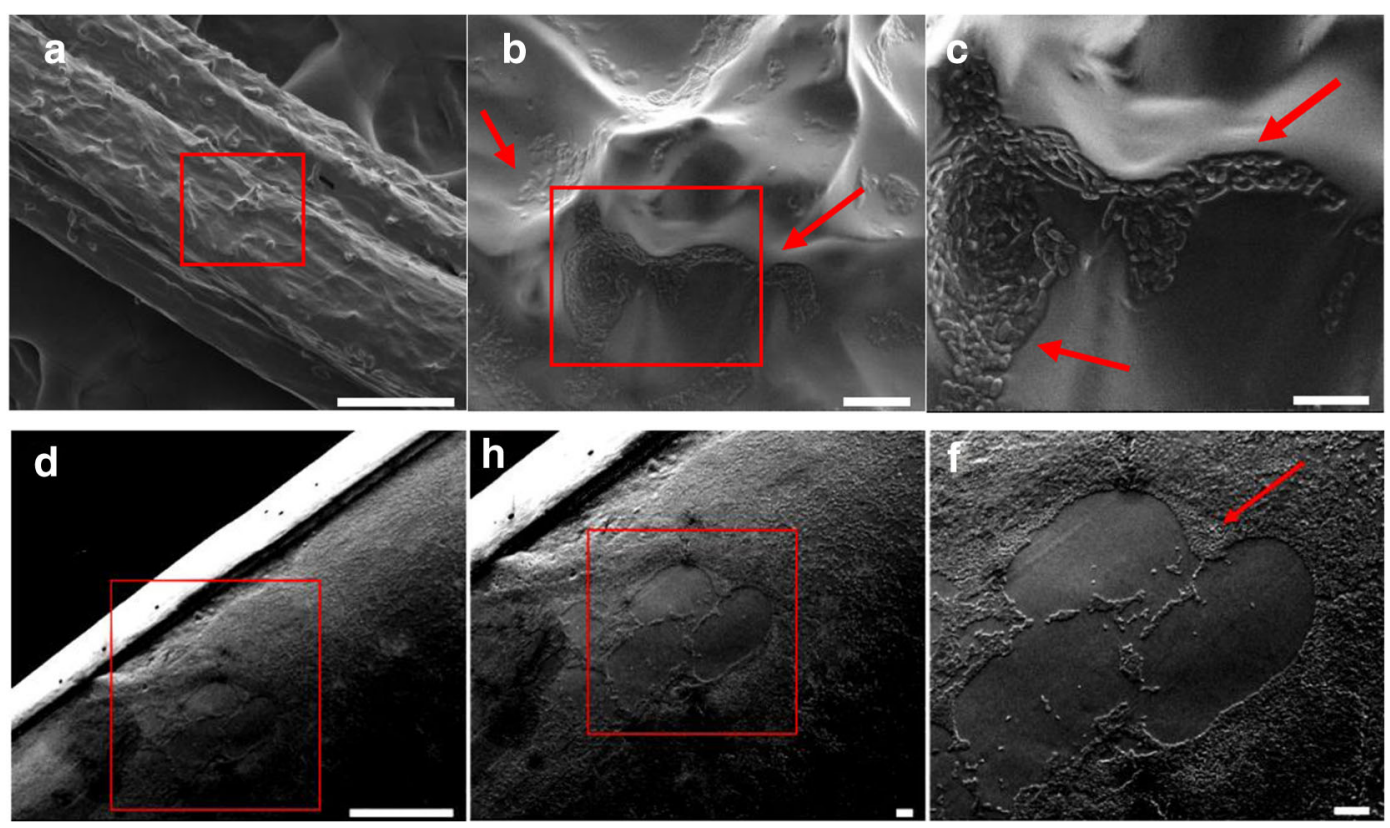

Fig. 5 Rs colonizes root and structureless replicas A root replica (A-C) and a flat needle replica (D-F) were inoculated with $R s$ bacteria and visualized using SEM. Bacteria cluster in valleys on the root replica, but no specific colonization region is observed on the needle replica. Red boxes $=$ region shown in the next image. Arrows point to bacteria. Scale bars: A, D = $100 \mu \mathrm{m}, \mathrm{B}, \mathrm{C}, \mathrm{E}, \mathrm{F}=$ $10 \mu \mathrm{m}$ difficult to separate from all other chemical and molecular mechanisms governing this interaction. When synthetic systems were used to isolate the role of leaf surface microstructure in leaf-microorganism interaction, new mechanisms of behavior were observed (Doan and Leveau 2015; Sirinutsomboon et al. 2011).

Transferring such a synthetic system to roots to study the effect of root surface microstructure in root environment interactions is challenging due to root-specific properties such as delicacy and curvature. Previous studies replicated a root cross section, with emphasis on the hierarchical structure of the cross section, with nanometer resolution, to generate a porous membrane (Van Opdenbosch et al. 2011; Yongjum et al. 2016). In those studies the original tissue was hydrolyzed from silicon or a similar material based coating from to generate a thin membrane that mimicked the hierarchical structure of a root cross section. However, no such work was performed on the root surface microstructure.

Here we used biomimetics to replicate root surface microstructure, but another way of examining the effect of root surface microstructure is through $3 \mathrm{D}$ printing technology. 3D printing allows a range of materials and resolutions previously unobtained (Ngo et al. 2018). However, using 3D printing to accurately replicate root microstructure requires specialized tools such as microCT, which can be challenging. Additionally, both microCT and high-resolution 3D printers are expensive machines that are not always available to researchers.

Here we adapt tools from previously reported soft lithography of leaf surfaces to the surface of a root. This method is widely available, requires no expensive machinery and can be performed in any laboratory. We predict that this new system can address research questions from a novel and unique perspective. Any interaction between the root and its environment, specifically interactions with microorganisms, can be viewed using this novel system. This allows researchers to emphasize the structural component in the interaction, discovering new elements of this interaction. This method should be broadly applicable to others working on such interactions.

Acknowledgments We would like to thank Dr. Einat Zelinger and the Center for Scientific Imaging (CSI) at the Faculty of Agriculture, Hebrew University, Rehovot for their assistance with SEM imaging. Research was supported by seed funds from The Agricultural Research Organization to MK and the Foundation for Food and Agriculture Research and Hatch Funds (IND 1013630) to AIP. 
Open Access This article is licensed under a Creative Commons Attribution 4.0 International License, which permits use, sharing, adaptation, distribution and reproduction in any medium or format, as long as you give appropriate credit to the original author(s) and the source, provide a link to the Creative Commons licence, and indicate if changes were made. The images or other third party material in this article are included in the article's Creative Commons licence, unless indicated otherwise in a credit line to the material. If material is not included in the article's Creative Commons licence and your intended use is not permitted by statutory regulation or exceeds the permitted use, you will need to obtain permission directly from the copyright holder. To view a copy of this licence, visit http://creativecommons.org/licenses/by/4.0/.

\section{References}

Anderson JR et al (2000) Fabrication of Microfluidic Systems in Poly (dimethylsiloxane). Electrophoresis 21(1):27-40 https://www.researchgate.net/profile/Hongkai $\mathrm{Wu} /$ publication/227935955_Fabrication_of_microfluidic systems_in_polydimethylsiloxane/links/55800ca80 $\overline{8}$ aec87640df1c7f.pdf (September 12, 2017)

Barthlott W, Mail M, Bhushan B, Koch K (2017) Plant surfaces: structures and functions for biomimetic innovations. NanoMicro Letters 9(2):23. https://doi.org/10.1007/s40820-0160125-1

Bhattacharjee A, Khan M, Kleiman M, Hochbaum AI (2017) Effects of growth surface topography on bacterial signaling in Coculture biofilms. ACS Appl Mater Interfaces 9(22): 18531-18539

Bhushan B (2009) Biomimetics: Lessons from Nature-an Overview. Philosophical Transactions of the Royal Society A 367:1445-1486 http://rsta.royalsocietypublishing. org/content/367/1893/1445.short (September 12, 2017)

Bixler GD, Bhushman B (2012) Biofouling : Lessons from Nature. Philosophical Transactions of the Royal Society A 370:2381-2417

Caldwell D, Kim BS, Iyer-Pascuzzi AS (2017) Ralstonia Solanacearum differentially colonizes roots of resistant and susceptible tomato plants. Phytopathology 107(5):528-536

Cormack RGH (1949) The development of root hairs in angiosperms. Bot Rev 15(9):583-612

Dalsing BL, Allen C (2014) Nitrate Assimilation Contributes to Ralstonia Solanacearum Root Attachment, Stem Colonization, and Virulence. J Bacteriol 196(5):949-960 http://jb.asm.org/content/196/5/949.short (September 12, 2017)

Dasgupta $\mathrm{N}$ et al (2015) Nanotechnology in agro-food: from fi Eld to plate. Frin 69:381-400. https://doi.org/10.1016/j. foodres.2015.01.005

Diem HG (1974) Micro-organisms of the leaf surface: estimation of the Mycoflora of the barley Phyllosphere. J Gen Microbiol 80(1):77-83

Doan HK, Leveau JHJ (2015) Artificial surfaces in Phyllosphere microbiology. Phytopathology 105(8):1036-1042

French E, Kim BS, Rivera-Zuluaga K, Iyer-Pascuzzi AS (2018) Whole root Transcriptomic analysis suggests a role for Auxin pathways in resistance to Ralstonia Solanacearum in tomato. Mol Plant Microbe Interact 31(4):432-444

Gule NP, Begum NM, Klumperman B (2016) Advances in biofouling mitigation: a review. Crit Rev Environ Sci Technol 46(6):535-555

Kang Y et al (2002) Ralstonia Solanacearum Requires Type 4 Pili to Adhere to Multiple Surfaces and for Natural Transformation and Virulence. Mol Microbiol 46(2):427437. https://doi.org/10.1046/j.1365-2958.2002.03187.x/full (September 12, 2017)

Kerai LV, Hilton S, Murdan S (2015) UV-curable gel formulations: potential drug carriers for the topical treatment of nail diseases. Int J Pharm 492(1-2):177-190

Kim BS et al (2016) Bacterial Wilt Disease: Host Resistance and Pathogen Virulence Mechanisms. Physiological and Molecular Plant Pathology 95:37-43 http://www. sciencedirect.com/science/article/pii/S0885576516300182 (September 12, 2017)

Kleiman M, Brubaker KS, Nguyen DT, Esser-Kahn AP (2015) Bio-inspired morphogenesis using microvascular networks and reaction-diffusion. Chem Mater 27(13):4871-4876. https://doi.org/10.1021/acs.chemmater.5b01947

Koch K, Bhushan B, Jung YC, Barthlott W (2009) Fabrication of artificial Lotus leaves and significance of hierarchical structure for Superhydrophobicity and low adhesion. Soft Matter 5(7):1386-1393

Koch, K, B Bhushan, and W Barthlott. 2010. "Multifunctional Plant Surfaces and Smart Materials." In Springer Handbook of Nanotechnology, , 1399-1436. https://doi.org/10.1007 /978-3-642-02525-9_41 (September 12, 2017)

Lai BY et al (2009) Designing Superhydrophobic porous nanostructures with tunable water adhesion. Adv Mater 21:37993803

Lau KKS et al (2003) Superhydrophobic carbon nanotube forests. Nano Lett 3:1701

Leitner D, Klepsch S, Ptashnyk M, Marchant A, Kirk GJ, Schnepf A, Roose T (2010) A dynamic model of nutrient uptake by root hairs. New Phytol 185(3):792-802. https://doi. org/10.1111/j.1469-8137.2009.03128.x/full

Lindsey BI, Pugh GJF (1976) Distribution of Microfungi over Surfaces of Attached Leaves of Hippophae rhamnoides. Transactions of the British Mycological Society 67(DEC): $427-433$

Liu T et al (2007) Corrosion behavior of super-hydrophobic surface on copper in seawater. Electrochim Acta 52(28):80038007

Mancuso, Stefano. 2012. Measuring roots: an updated approach

Mark, JE, HR Allcock, and R West. 2005. Inorganic Polymers. https://www.google.com/books?hl=en \&lr=\&id=7 Y y b TrluK B g C \& o i = f n d \& p g = P R $9 \&$ d q $=$ inorganic + polymers \& ots $=5 \mathrm{pVp} 9 \mathrm{C} 8 \mathrm{nGT} \& \operatorname{sig}=4 \mathrm{Po}_{-}$ QVSeIBfmtPZAJEP3AeOE2V0 (September 12, 2017)

Marschner, Horst. 2011. Marschner's mineral nutrition of higher plants

Mata A, Fleischman AJ, Roy S (2005) Characterization of Polydimethylsiloxane (PDMS) Properties for Biomedical Micro/nanosystems. Biomed Microdevices 7(4):281-293. https://doi.org/10.1007/s10544-005-6070-2 (September 12, 2017) 
Ngo TD et al (2018) Additive manufacturing (3D printing): a review of materials, methods, applications and challenges. Compos Part B 143:172-196

Nion YA, Toyota K (2015) Recent Trends in Control Methods for Bacterial Wilt Diseases Caused by Ralstonia Solanacearum. Microbes and Environments 30(1):1-11 https://www.jstage. jst.go.jp/article/jsme2/30/1/30_ME14144/_article/-char/ja/ (September 12, 2017)

Opdenbosch V, Daniel GF-P, Paris O, Zollfrank C (2011) Silica replication of the hierarchical structure of wood with nanometer precision. J Mater Res 26(10):1193-1202

Paretkar D, Xu X, Hui CY, Jagota A (2014) Flattening of a patterned compliant solid by surface stress. Soft Matter 10(23):4084-4090

Peng CW et al (2013) UV-curable Nanocasting technique to prepare bio-mimetic super-hydrophobic non-fluorinated polymeric surfaces for advanced anticorrosive coatings. Polym Chem 4(4):926-932

Popham RA, Henry RD (1955) Multicellular root hairs on adventitious roots of Kalanchoe Fedtschenkoi. Ohio J Sci 55(5): 301-307

Rogers JA, Nuzzo RG (2005) Recent Progress in Soft Lithography. Mater Today 8(2):50-56 http://www. sciencedirect.com/science/article/pii/S1369702105007029 (September 12, 2017)

Schneider F, Draheim J, Kamberger R, Wallrabe U (2009) Process and Material Properties of Polydimethylsiloxane (PDMS) for Optical MEMS. Sensors and Actuators A: Physical 151(2):9599 http://www.sciencedirect.com/science/article/pii/S0924 424709000466 (September 12, 2017)

Sirinutsomboon B, Delwiche MJ, Young GM (2011) Attachment of Escherichia Coli on plant surface structures built by microfabrication. Biosyst Eng 108(3):244-252

Smith S, De Smet I (2012) Root system architecture: insights from Arabidopsis and cereal crops. Philosophical Transactions of the Royal Society B: Biological Sciences 367(1595):14411452. https://doi.org/10.1098/rstb.2011.0234

Solga A, Cerman Z, Striffler BF, Spaeth M (2007) The Dream of Staying Clean: Lotus and Biomimetic Surfaces. Bioinspir Biomim 2(4):S126. https://doi.org/10.1088/1748-3182/2/4 /S02/meta (September 12, 2017)

Szyndler MW, Haynes KF, Potter MF, Corn RM, Loudon C (2013) Entrapment of bed bugs by leaf Trichomes inspires microfabrication of biomimetic surfaces. J R Soc Interface 10(83):20130174. https://doi.org/10.1098/rsif.2013.0174

Thieme BM et al (2001) Generation of Ultrahydrophobic Properties of Aluminium A First Step to Self-Cleaning Transparently Coated Metal Surfaces. AdvEng Mater 9: 691-695
Tricinci $\mathrm{O}$ et al (2015) 3D micropatterned surface inspired by Salvinia Molesta via direct laser lithography. ACS Appl Mater Interfaces 7:25560

Tsai SL, Harris PJ, Lovell PH (2004) Bands of root hairs are produced in tomato (Lycopersicon Esculentum) in response to specific combinations of Thermoperiods and photoperiods. N Z J Crop Hortic Sci 32(1):121-129

Weng C-j et al (2011) Advanced anticorrosive coatings prepared from the mimicked Xanthosoma Sagittifolium-leaf-like Electroactive epoxy with synergistic effects of Superhydrophobicity and redox catalytic capability. Chem Mater 23:2075-2083

Yang T-i et al (2012) Synergistic effect of Electroactivity and hydrophobicity on the anticorrosion property of roomtemperature-cured epoxy coatings with multi-scale structures mimicking the surface of Xanthosoma Sagittifolium leaf $\uparrow$. Mater Chem 22:15845-15852

Yang WC, Lin YM, Cheng YS (2013) Ralstonia Solanacearum RSc0411 (lptC) Is a Determinant for Full Virulence and Has a Strain-Specific Novel Function in the T3SS Activity. Microbiology 159(6):1136-1148. https://doi.org/10.1099 /mic.0.064915-0 (September 12, 2017)

Yang Y et al (2018) 3D-printed biomimetic super-hydrophobic structure for microdroplet manipulation and oil / water separation. Adv Mater 9:1704912

Yongjum M et al (2016) Synthesis of hierarchical ordered porous functional materials using willow wickers as templates for recyclable photo-catalytic applications. J Porous Mater 23: 225-230

Zhang F et al (2011) Preparation of Superhydrophobic films on titanium as effective corrosion barriers. Appl Surf Sci 257(7): $2587-2591$

Zhang B, Luo Y, Pearlstein AJ, Aplin J, Liu Y, Bauchan GR, Payne GF, Wang Q, Nou X, Millner PD (2014) Fabrication of biomimetically patterned surfaces and their application to probing plant-Bacteria interactions. ACS Appl Mater Interfaces 6(15):12467-12478

Zhao X-m, Xia Y, Whitesides GM (1997) Soft lithographic methods for Nano-fabrication. J Mater Chem 7:1069-1074

Zhao BN et al (2007) A novel ultra-hydrophobic surface : statically non-wetting but dynamically non-sliding **. Adv Funct Mater 17:2739-2745

Publisher's note Springer Nature remains neutral with regard to jurisdictional claims in published maps and institutional affiliations. 